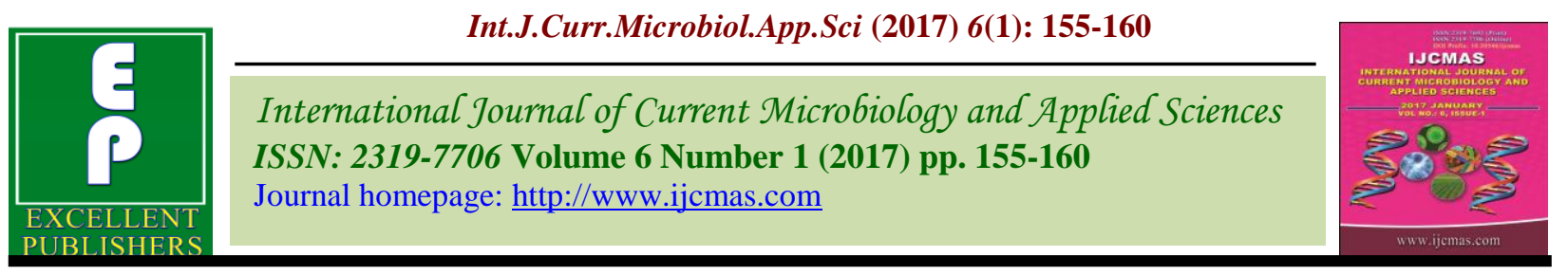

Original Research Article

http://dx.doi.org/10.20546/ijcmas.2017.601.019

\title{
Prevalence and Antibacterial Susceptibility Pattern of Aerobic Bacterial Pathogens isolated from Pus-A Retrospective Study from a Teaching Hospital in Tamilnadu, India
}

\author{
P. Shankar ${ }^{1 *}$ and M. Kavitha ${ }^{2}$ \\ ${ }^{1}$ Department of Microbiology, Government Villupuram Medical College, Tamilnadu, India \\ ${ }^{2}$ Department of Microbiology, Government Kilpauk Medical College, Tamilnadu, India \\ *Corresponding author
}

\section{A B S T R A C T}

Keywords

Wound

infection, pus,

antibiogram,

resistance,

Aerobic

Bacterial

Pathogens

Article Info

Accepted:

12 December 2016

Available Online:

10 January 2017
The objective of the present study was to ascertain the prevalence of pathogens of infective wounds and to study the antimicrobial resistance among them with commonly used antibiotics retrospectively. A total of 912 pus specimens from patients were received from IPDs and OPDs and tested for aerobic culture and sensitivity in Villupuram Medical College, Tamilnadu, India, were cultured by standard microbiological procedures for the isolation of pathogens. The isolates were subjected to antimicrobial susceptibility assay to investigate the resistance pattern of isolates by disc diffusion method. A total of 912 specimens were collected, of which 528/912 (57.9\%) were found to be positive for pus culture and 384/912 (42.1\%) showed no growth. The positive samples were from both 305/528 (57.8\%) male and 223/528 $(42.2 \%)$ female patients of various age groups. In the present study 530 isolates were obtained from the culture positive pus $(n=528)$ specimens comprising seven different bacterial pathogens and nine different species were reported. In the present study the antibiogram of Gram positive cocci showed higher resistance rate of all the antibacterials used in this study.

\section{Introduction}

Wound infections are generally inflammation, usually with pus formation, in most cases which are caused by one of the pyogenic bacteria which includes Staphylococcus aureus, Pseudomonas sp., Klebsiella spp., E. coli, etc., and which results the accumulation of dead leukocytes and infectious agent generally known as pus(Bowler et al., 2001). Wound infections cause a significant morbidity and mortality and also which is considered to be one of the most important and common hospital acquired infections worldwide. Infection in wound can have an adverse impact on the patient's quality of life and also infected wounds are likely to be more painful, hypersensitive and odorous, resulting in increased discomfort and inconvenience for the patient (Kotz et al., 2009).

The dissemination of multi-drug resistant bacterial pathogens have made it important to the problem of wound infections. This is 
particularly worse in countries where sale of antibiotics is under poor control (Sara Reardon). The culture of wound swab is the most often used method of identification of wound infection and these cultures provide useful data to the better diagnostic and therapeutic decision making (Bonham et al., 2009). The main objectives of the present study was to ascertain the distribution of aerobic pathogenic bacteria from pus and the susceptibility pattern of antibacterial agents used.

\section{Materials and Methods}

\section{Study subjects}

A total of 912pus specimens from patients were received from IPDs and OPDs and tested for aerobic culture and sensitivity in Villupuram Medical College, Tamilnadu, India, and the reports were analyzed retrospectively over the period of eleven months from May 2015 to March 2016.

\section{Processing of Specimen}

Pus specimens were cultured according to standard microbiological procedures. The isolates were identified using routine bacteriological procedures by colonymorphology, cultural and biochemical methods and subjected to antibacterial assay.

\section{Determination of antibiotic susceptibility}

Antibiotic susceptibility testing was carried out by Kirby-Bauer disc diffusion assay on Muller Hinton agar (HiMedia) to determine the antimicrobial susceptibility profiles of pathogenic isolatesaccording to Clinical and Laboratory Standards Institute (CLSI) guidelines. The panel of antibiotic disks (HiMedia) include aminoglycoside

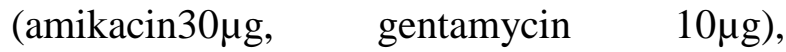
penicillins(Co-amoxyclav10 $\mu \mathrm{g}, \quad$ amoxicillin $10 \mu \mathrm{g}$, penicillin $10 \mu \mathrm{g}$,), fluoroquinolones (ciprofloxacin $5 \mu \mathrm{g}$ ), macrolides (erythromycin $15 \mu \mathrm{g}$ ), cephalosporins (cefotaxime $30 \mu \mathrm{g}$ ) other antibiotic include cotrimaxazole (1.25/23.75 $\mu \mathrm{g}) \quad$ and doxycycline $30 \mu \mathrm{g})$. The diameter of the zone of inhibition produced by each antibiotic disk was measured and the result was interpreted as susceptible or resistance to the antibiotics used and the zone of inhibition was compared with the standard chart provided by the manufacturer of the antibiotic disks.

\section{Results and Discussion}

\section{Distribution of pathogens from pus}

A total of 912 specimens were collected in a period of eleven months from May 2015 to March 2016,of which 528/912 (57.9\%) were found to be positive for pus culture and $384 / 912$ (42.1\%) showed no growth. The positive samples were from both $305 / 528$ (57.8\%) male and 223/528 (42.2\%) female patients of various age groups (Table 1).In the present study530 isolates were obtained from the culture positive pus $(n=528)$ specimens comprising seven different bacterial pathogens and nine different species were reported, of which 392/530 (74\%) were found to be Gram negative bacilli and 138/530 (26\%) were found to be Gram positive cocci. Amongst 530isolates, Staphylococcus aureus (119 (22.5\%)) was the predominant pyogenic bacterial pathogen isolated in our study, followed by 115 (21.7\%) were Pseudomonas spp., 110 (20.7\%) were Klebsiella spp., 106 (20\%) were E. coli, 45(8.5\%) were Proteus spp., 19(3.6\%) were coagulase negative Staphylococcus spp.(CoNS), 16(3\%) were reported as Acenetobacter sp. (Table 2).

\section{Antibacterial susceptibility testing}

Of the 392/530Gram negative bacillusisolates from pus weretested by Kirby-Bauer disc diffusion assay against a panel of antibiotics such asamikacin, amoxycillin, ciprofloxacin, 
cotrimaxazole and cefotaxime. Pseudomonas sp. $(\mathrm{n}=115)$ showed 45/115 (39\%), 115/115 (100\%), 63/115 (55\%), 103/115 (90\%), 51/115 (44\%) resistance, Klebsiella pneumoniae $(\mathrm{n}=61)$ showed 17/61 (28\%), 61/61 (100\%), 40/61 (66\%), 46/61 (75\%), 46/61 (75\%) resistance, Klebsiella oxytoca $(\mathrm{n}=49)$ showed 24/49 (49\%), 47/49 (56\%), 39/49 (80\%), 43/49 (88\%), 39/49 (80\%) resistance, E.coli $(\mathrm{n}=106)$ showed $25 / 106$ (24\%), 98/106 (93\%), 75/106 (71\%), 86/106 (81\%), 71/106 (67\%) resistance, Proteus mirabilis $(\mathrm{n}=29)$ showed 16/29 $(55 \%), 23 / 29$ (79\%), 14/29 (48\%), 16/55 (81\%), 16/29 (55\%) resistance, Proteus vulgaris $(\mathrm{n}=16)$ showed 7/16 (44\%), 16/16 (100\%), 8/16 (50\%), 14/16 (87\%), 9/16 (56\%) resistance and Acenetobacter sp. $(\mathrm{n}=16)$ showed $7 / 16$ (44\%), 16/16 (100\%), 4/16 (25\%), 11/16 (69\%), 11/16 (69\%) resistance against the panel of antibiotics respectively(Table 3).
Of the 138/530 Gram positive isolates from pus were tested against a panel of antibiotics such as amikacin, co-amoxyclav, doxycycline, gentamycin, penicillin, erythromycin, Staphylococcus aureus $(\mathrm{n}=119)$ showed 29/119 (24\%), 54/119 (45\%), 25/119 (21\%), 19/119 (16\%), 109/119 (92\%), 68/119 $(57 \%)$ resistance against the panel of antibiotics respectively, CoNS $(\mathrm{n}=19)$ showed 8/19 (42\%),16/19 (84\%), 5/19 (26\%), 9/19 (47\%), 16/19 (84\%), 9/19 (47\%) resistance to the panel of antibiotics respectively (Table 4).

In the present study we found that prevalence of Gram negative bacillus was higher than Gram positive bacteria isolated in our study. Our findings are correlated with a study conducted in North India in 2009 (3) Staphylococcus aureus 119/530 (22.5\%) was found to be highest prevalence of all the organisms isolated from pus (fig 1).

Table.1 Sex wise distribution of culture positive pus samples

\begin{tabular}{|c|c|}
\hline Sex & $\begin{array}{c}\text { No. of culture positive pus(n) } \\
\mathbf{n}=(\mathbf{5 2 8})\end{array}$ \\
\hline Male & $305(57.8 \%)$ \\
\hline Female & $223(42.2 \%)$ \\
\hline Total & 528 \\
\hline
\end{tabular}

Table.2 Distribution of bacterial pathogens isolated from pus

\begin{tabular}{|c|c|}
\hline Pathogens & $\begin{array}{c}\text { No. of Isolates } \\
\text { n }(\%)\end{array}$ \\
\hline Staphlococcus aureus & $119(22.5 \%)$ \\
\hline Pseudomonas sp. & $115(21.7 \%)$ \\
\hline Klebsiella spp. & $110(20.7 \%)$ \\
\hline E. coli & $106(20 \%)$ \\
\hline Proteus spp. & $45(8.5 \%)$ \\
\hline CoNS & $19(3.6 \%)$ \\
\hline Acenetobacter sp. & $16(3 \%)$ \\
\hline Total & 530 \\
\hline
\end{tabular}


Table.3 Resistance pattern of Gram negative bacilli isolated from pus

\begin{tabular}{|c|c|c|c|c|c|c|c|c|c|c|c|}
\hline & \multicolumn{2}{|c|}{ AK } & \multicolumn{2}{|c|}{ AMX } & \multicolumn{2}{|c|}{ CIP } & \multicolumn{2}{|c|}{ COT } & \multicolumn{2}{|c|}{ CTX } \\
\hline & & $\mathbf{S}$ & $\mathbf{R}$ & $\mathbf{S}$ & $\mathbf{R}$ & $\mathbf{S}$ & $\mathbf{R}$ & $\mathbf{S}$ & $\mathbf{R}$ & $\mathbf{S}$ & $\mathbf{R}$ \\
\hline \multicolumn{2}{|c|}{$\begin{array}{l}\text { Pseudomonas } \\
\text { aeruginosa }(\mathrm{n}=115)\end{array}$} & $\begin{array}{c}70 \\
(61 \%)\end{array}$ & $\begin{array}{c}45 \\
(39 \%)\end{array}$ & 0 & $\begin{array}{c}115 \\
(100 \%)\end{array}$ & $\begin{array}{c}52 \\
(45 \%)\end{array}$ & $\begin{array}{c}63 \\
(55 \%) \\
\end{array}$ & $\begin{array}{c}12 \\
(10 \%)\end{array}$ & $\begin{array}{c}103 \\
(90 \%)\end{array}$ & $\begin{array}{c}64 \\
(56 \%)\end{array}$ & $\begin{array}{c}51 \\
(44 \%)\end{array}$ \\
\hline \multirow{2}{*}{$\begin{array}{l}\text { Klebsiella } \\
(\mathrm{n}=110)\end{array}$} & $\begin{array}{l}\text { pneumoniae } \\
(\mathrm{n}=61)\end{array}$ & $\begin{array}{c}44 \\
(72 \%) \\
\end{array}$ & $\begin{array}{c}17 \\
(28 \%) \\
\end{array}$ & 0 & $\begin{array}{c}61 \\
(100 \%)\end{array}$ & $\begin{array}{c}21 \\
(34 \%)\end{array}$ & $\begin{array}{c}40 \\
(66 \%) \\
\end{array}$ & $\begin{array}{c}15 \\
(25 \%) \\
\end{array}$ & $\begin{array}{c}46 \\
(75 \%) \\
\end{array}$ & $\begin{array}{c}15 \\
(25 \%)\end{array}$ & $\begin{array}{c}46 \\
(75 \%) \\
\end{array}$ \\
\hline & $\begin{array}{l}\text { oxytoca } \\
(\mathrm{n}=49)\end{array}$ & $\begin{array}{c}25 \\
(51 \%)\end{array}$ & $\begin{array}{c}24 \\
(49 \%)\end{array}$ & $\begin{array}{c}2 \\
(4 \%)\end{array}$ & $\begin{array}{c}47 \\
(56 \%)\end{array}$ & $\begin{array}{c}10 \\
(20 \%)\end{array}$ & $\begin{array}{c}39 \\
(80 \%)\end{array}$ & $\begin{array}{c}6 \\
(12 \%)\end{array}$ & $\begin{array}{c}43 \\
(88 \%)\end{array}$ & $\begin{array}{c}10 \\
(20 \%)\end{array}$ & $\begin{array}{c}39 \\
(80 \%)\end{array}$ \\
\hline \multicolumn{2}{|c|}{ E. $\operatorname{coli}(\mathrm{n}=106)$} & $\begin{array}{c}81 \\
(76 \%) \\
\end{array}$ & $\begin{array}{c}25 \\
(24 \%) \\
\end{array}$ & $\begin{array}{c}8 \\
(7 \%) \\
\end{array}$ & $\begin{array}{c}98 \\
(93 \%) \\
\end{array}$ & $\begin{array}{c}31 \\
(29 \%)\end{array}$ & $\begin{array}{c}75 \\
(71 \%) \\
\end{array}$ & $\begin{array}{c}20 \\
(19 \%) \\
\end{array}$ & $\begin{array}{c}86 \\
(81 \%) \\
\end{array}$ & $\begin{array}{c}35 \\
(33 \%) \\
\end{array}$ & $\begin{array}{c}71 \\
(67 \%) \\
\end{array}$ \\
\hline \multirow{2}{*}{$\begin{array}{l}\text { Proteus } \\
(\mathrm{n}=45)\end{array}$} & $\begin{array}{l}\text { mirabilis } \\
(\mathrm{n}=29)\end{array}$ & $\begin{array}{c}13 \\
(45 \%)\end{array}$ & $\begin{array}{c}16 \\
(55 \%)\end{array}$ & $\begin{array}{c}6 \\
(21 \%)\end{array}$ & $\begin{array}{c}23 \\
(79 \%)\end{array}$ & $\begin{array}{c}15 \\
(52 \%)\end{array}$ & $\begin{array}{c}14 \\
(48 \%)\end{array}$ & $\begin{array}{c}13 \\
(45 \%)\end{array}$ & $\begin{array}{c}16 \\
(55 \%)\end{array}$ & $\begin{array}{c}13 \\
(45 \%)\end{array}$ & $\begin{array}{c}16 \\
(55 \%)\end{array}$ \\
\hline & $\begin{array}{l}\text { vulgaris } \\
(\mathrm{n}=16)\end{array}$ & $\begin{array}{c}9 \\
(56 \%)\end{array}$ & $\begin{array}{c}7 \\
(44 \%)\end{array}$ & 0 & $\begin{array}{c}16 \\
(100 \%)\end{array}$ & $\begin{array}{c}8 \\
(50 \%)\end{array}$ & $\begin{array}{c}8 \\
(50 \%)\end{array}$ & $\begin{array}{c}2 \\
(13 \%)\end{array}$ & $\begin{array}{c}14 \\
(87 \%)\end{array}$ & $\begin{array}{c}7 \\
(44 \%)\end{array}$ & $\begin{array}{c}9 \\
(56 \%)\end{array}$ \\
\hline \multicolumn{2}{|c|}{$\begin{array}{l}\text { Acenetobacter sp. } \\
(\mathrm{n}=16)\end{array}$} & $\begin{array}{c}9 \\
(56 \%)\end{array}$ & $\begin{array}{c}7 \\
(44 \%)\end{array}$ & 0 & $\begin{array}{c}16 \\
(100 \%)\end{array}$ & $\begin{array}{c}12 \\
(75 \%)\end{array}$ & $\begin{array}{c}4 \\
(25 \%)\end{array}$ & $\begin{array}{c}5 \\
(31 \%)\end{array}$ & $\begin{array}{c}11 \\
(69 \%)\end{array}$ & $\begin{array}{c}5 \\
(31 \%)\end{array}$ & $\begin{array}{c}11 \\
(69 \%)\end{array}$ \\
\hline
\end{tabular}

AK=amikacin, AMX=amoxicillin, CIP=ciprofloxacin, COT=cotrimaxazole, CTX=cefotaxime.

Table.4 Resistance pattern of Gram positive cocci isolated from pus

\begin{tabular}{|c|c|c|c|c|c|c|c|c|c|c|c|c|}
\hline & \multicolumn{2}{|c|}{ AK } & \multicolumn{2}{|c|}{ AMC } & \multicolumn{2}{|c|}{ DO } & \multicolumn{2}{|c|}{$\mathbf{G}$} & \multicolumn{2}{|c|}{$\mathbf{P}$} & \multicolumn{2}{|c|}{$\mathbf{E}$} \\
\hline & $\mathbf{S}$ & $\mathbf{R}$ & $\mathbf{S}$ & $\mathbf{R}$ & $\mathbf{S}$ & $\mathbf{R}$ & $\mathbf{S}$ & $\mathbf{R}$ & $\mathbf{S}$ & $\mathbf{R}$ & $\mathbf{S}$ & $\mathbf{R}$ \\
\hline $\begin{array}{l}\text { Staphlococcus } \\
\text { aureus } \\
(n=119)\end{array}$ & $\begin{array}{c}90 \\
(76 \%)\end{array}$ & $\begin{array}{c}29 \\
(24 \%)\end{array}$ & $\begin{array}{c}65 \\
(55 \%)\end{array}$ & $\begin{array}{c}54 \\
(45 \%)\end{array}$ & $\begin{array}{c}94 \\
(79 \%)\end{array}$ & $\begin{array}{c}25 \\
(21 \%)\end{array}$ & $\begin{array}{c}100 \\
(84 \%)\end{array}$ & $\begin{array}{c}19 \\
(16 \%)\end{array}$ & $\begin{array}{c}10 \\
(8 \%)\end{array}$ & $\begin{array}{c}109 \\
(92 \%)\end{array}$ & $\begin{array}{c}51 \\
(43 \%)\end{array}$ & $\begin{array}{c}68 \\
(57 \%)\end{array}$ \\
\hline CoNS $(n=19)$ & $\begin{array}{c}11 \\
(58 \%)\end{array}$ & $\begin{array}{c}8 \\
(42 \%)\end{array}$ & $\begin{array}{c}3 \\
(16 \%)\end{array}$ & $\begin{array}{c}16 \\
(84 \%)\end{array}$ & $\begin{array}{c}14 \\
(74 \%)\end{array}$ & $\begin{array}{c}5 \\
(26 \%)\end{array}$ & $\begin{array}{c}10 \\
(53 \%)\end{array}$ & $\begin{array}{c}9 \\
(47 \%)\end{array}$ & $\begin{array}{c}3 \\
(16 \%)\end{array}$ & $\begin{array}{c}16 \\
(84 \%)\end{array}$ & $\begin{array}{c}10 \\
(53 \%)\end{array}$ & $\begin{array}{c}9 \\
(47 \%)\end{array}$ \\
\hline
\end{tabular}

$\mathrm{AK}=$ amikacin, $\mathrm{AMC}=\mathrm{Co}$-amoxyclav, $\mathrm{DO}=$ doxycycline, $\mathbf{G}=$ gentamycin, $\mathbf{P}=$ penicillin, E=erythromycin

Fig 1

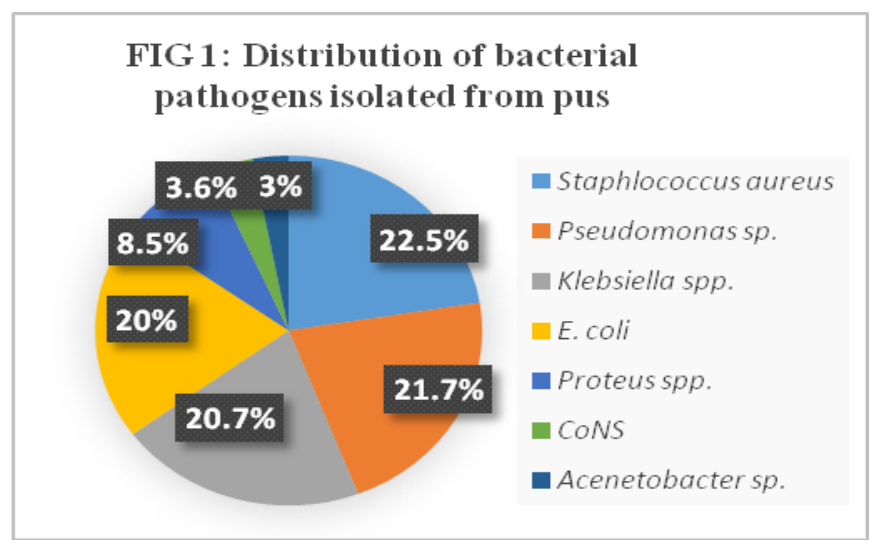


Fig 2

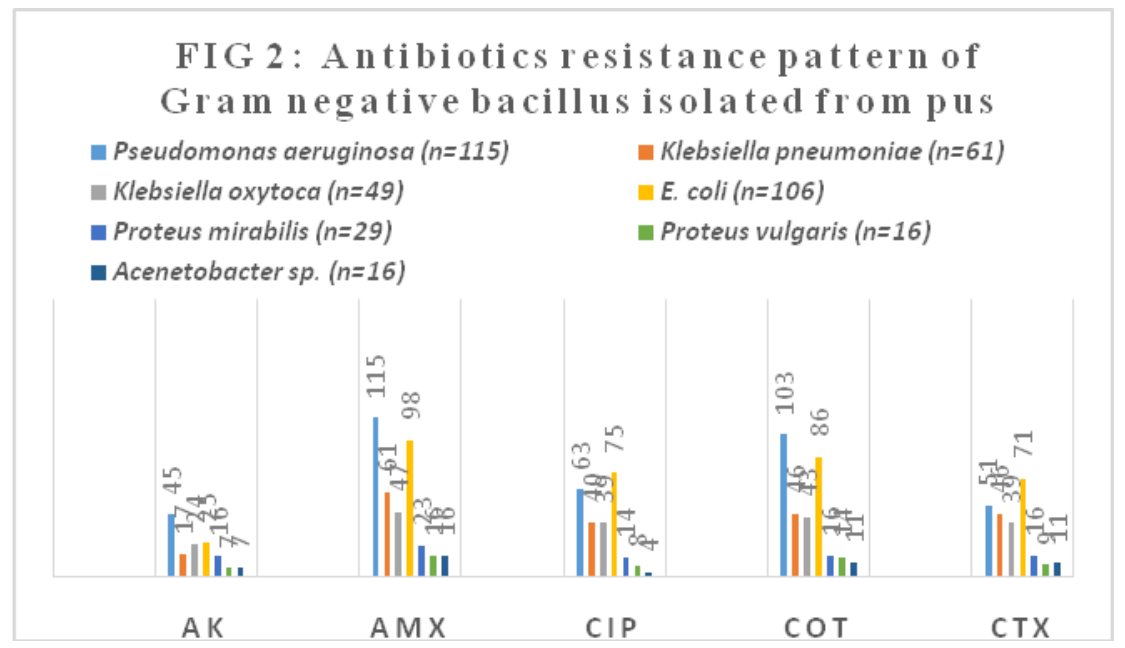

Fig 3

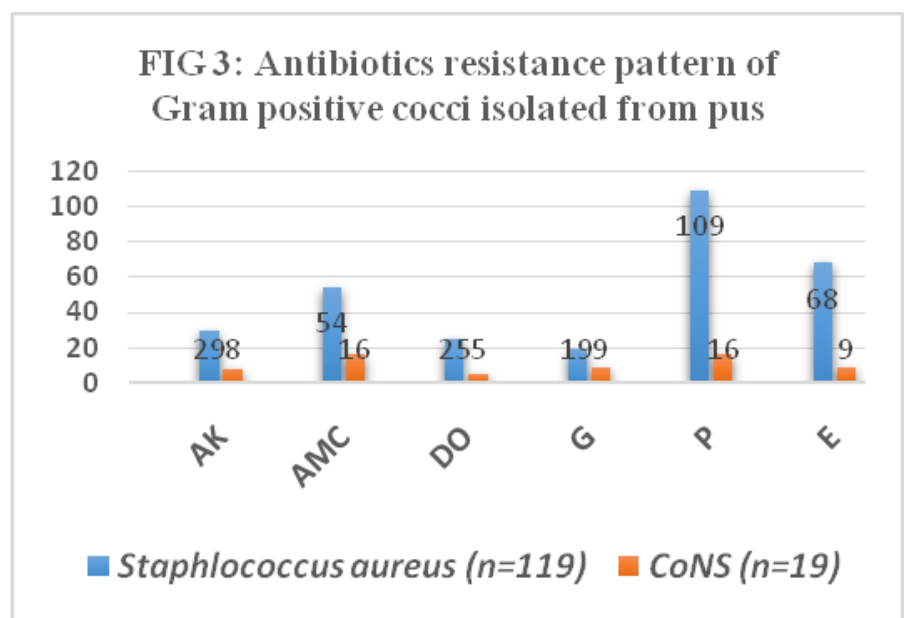

This finding was similar with other report in 2009 at Nigeria by Nwachukwu et al., (2009), other report by Agnihotri et al., (2004), shows that Pseudomonas aeruginosa were found to be highest incidence followed by Staphylococcus aureus which was not correlated with our findings. The antibiogram of Gram negative bacilli showed higher resistance rate of all the antibacterials used in this study. Pseudomonas aeruginosa and E. coli produced highest resistance against amoxicillin and cotrimaxazole and also E. coli showed higher resistance rates against ciprofloxacin and cefotaxime (fig 2). In the present study the antibiogram of Gram positive cocci showed higher resistance rate of all the antibacterials used in this study. In particular Staphylococcus aureus showed highest resistance rates to all the antimicrobials especially against penicillin and erythromycin (fig 3).

In conclusion, this study gives us an understanding to the current state of etiological agents of wound infections and their susceptibility pattern in our medical college hospital. Therefore accurate and rapid identification of antimicrobial resistance are necessary for the timely and accurate empirical therapy against wound infections. We suggest that good infection control practices and evidence based use of 
antibiotics will reduce the risk of higher degree of resistance against commonly used antibiotics and also will ensure that the spread of resistance will be under control.

\section{References}

Agnihotri, N., V. Gupta, R.M. Joshi. 2004. Aerobic bacterial isolate from burn wound infections and their anti biograms - a five-year study. Burns, 30p. 241.

Bonham, Phyllis, A. 2009. PhD, RN. Swab Cultures for Diagnosing Wound Infections: A Literature Review and Clinical Guideline. CWOCN, 36,4, p 389-395.

Bowler, P.G., B.I. Duerden and D.G. Armstrong. 2001. Wound Microbiology and Associated Approaches toWound Management. Clin. Microbiol. Rev., p. 244-269.

Clinical and Laboratory Standard Institute. 2012. Performance standards for antimicrobial susceptibility testing (M100-S22).

Ghosh, A., Karmakar, P.S., Pal, J., Chakrabor ty, N., Debnath, N.B., Mukherjee, J.D. 2009. Bacterial incidence and antibiotic sensitivity pattern in moderate and severe infections in hospitalised patients. J. Indian Med. Assoc., 107(1):21-2, 24-5.

Kotz, P., Fisher, J., McCluskey, P., Hartwell, S.D., Dharma, H. 2009. Use of a new silver barrier dressing, ALLEVYN Ag in exuding chronic wounds. Int. Wound J., 6:186 194.

Nwachukwu, N.C., Orji, F.A. and Okike, U.M. 2009. Antibiotic Susceptibility Patterns of Bacterial Isolates from Surgical Wounds in Abia State University Teaching Hospital (ABSUTH), Aba - Nigeria. Res. J. Medicine \& Med. Sci., 4(2): 575-579.

Sara Reardon. Antibiotic resistance sweeping developing world. Nature, 509, 141142.

\section{How to cite this article:}

Shankar, P., and Kavitha, M. 2017. Prevalence and Antibacterial Susceptibility Pattern of Aerobic Bacterial Pathogens isolated from Pus-A Retrospective Study from a Teaching Hospital in Tamilnadu. Int.J.Curr.Microbiol.App.Sci. 6(1): 155-160. doi: http://dx.doi.org/10.20546/ijcmas.2017.601.019 